\title{
The Software Testing Method Application and Research of Embedded Electronic System
}

\author{
Wei Yan-ping \\ NanChang Institute of Science \& Technology
}

\begin{abstract}
With the rapid development of computer hardware and software technology, the hardware size and performance of embedded system have been greatly improved, accordingly, the complexity and size of the embedded system software have also doubled. However, the particularity of the embedded system requires the hardware system and application software operated on it to be concise and efficient, stable and reliable. This makes the proportion of the software development in the whole embedded system development higher and higher. The software quality plays a crucial role in the whole product quality .As a result, we urgently need a testing method and tools for embedded field to improve software quality and reliability, shorten the software development period and reduce development costs. Under this background, this paper goes into the testing technology of embedded electronic system software and applies the research results to testing. From the actual needs, it goes into the software metrics technology and applies it to vehicle electronic system testing.
\end{abstract}

Keywords- Electronic system, embedded software, signal information selection, software testing

\section{BRIEF INTRODUCTION}

As an important branch of computer software, embedded software is applied more and more extensive in the fields of signal information selection, consumer electronics, transportation system, industrial control, medical equipment and military and so on. With the development of embedded technology, growing embedded application and increasing complexity of the embedded system, it demands the scale and complexity of embedded software to constantly improve. The quality and development period of the embedded software play a critical role to the final product quality and market time. The testing technology of embedded software becomes a hot spot of current research.

With the progress of computer hardware technology, the integration of hardware is higher and higher. This also greatly improves the hardware performance of the embedded devices; At the same time, the adoption of mature commercial operating system makes the system operate on a high-performance, reliable software platform and lays a good foundation to achieving a variety of large, complex application. The increasing system complexity naturally requires the corresponding powerful and stable application software, so the scale of software development in embedded system development is bigger and bigger. Meanwhile, the system requires the application to be concise and efficient, stable and reliable. It makes the software development time longer and longer in the whole system development. The software quality plays a crucial role in the whole product quality. However, because the software development is a lack of scientific management, the software developed is unable to get a good testing and analysis and the programs are delivered to users without effective testing. Those untested codes with potential risk are given to customers, often bring huge economic losses to the user, even endanger people's life in some special field.

Under this background, this paper is put forward, combined with the characteristics of the current embedded software and software testing theory, using mature software metrics technology and the improved testing model. It carries on detailed design of vehicle electronic system in stages of unit testing, integration testing, system testing and regression testing. It also makes thorough analysis to the testing results of vehicle electronic system in aspects of code review, memory leak, program performance.

\section{GPS RECEIVING PRINCIPLE}

GPS receiver can receive the time information accurate to nanosecond used for time service. It can be used for forecasting the general position of the satellites ephemeris in the next few months .It's used for calculating the broadcast ephemeris of the required satellite coordinates when positioning and GPS system information, such as satellite status. By measuring the GPS receiver match code can we get the distance from the satellite to the receiver. Since there is some error of the satellite receiver clock and atmospheric propagation, it's called pseudo range. The pseudo range for OA code is called UA code pseudo range, and the accuracy is about 20 meters. The pseudo range for $\mathrm{P}$ code is called $\mathrm{P}$ code pseudo range, and the accuracy is about 2 meters.

For the satellite signal GPS receiver received, it will decode or adopt other technology to remove the information modulated on the carrier in order to restore the carrier .Strictly speaking, carrier phase should be called carrier frequency phase. It's the phase difference between the satellite signal carrier phase position affected by Doppler shift and the signal phase caused by oscillation of the receiver itself .Generally we can record the the change value by measuring the epoch time set by receiver clock and keeping tracking the satellite signal. However, the initial value of the epoch phase of the receiver and satellite oscillator is unknown, and the phase position integer of the initial epoch is also unknown, namely ambiguity of the whole circle. We can only calculate them when processing data. The accuracy of the phase observed value can be as high as millimeter, with the premise of working out the whole circle ambiguity. As a result, the phase observed value can only be used on condition of relative positioning and 
continuous observation value .In order to achieve the positioning precision superior to meter, we can only use phase observation data.

Closely connected with the ground data center is the differential reference station. The GPS module of the differential reference station is linked to the CPU of the

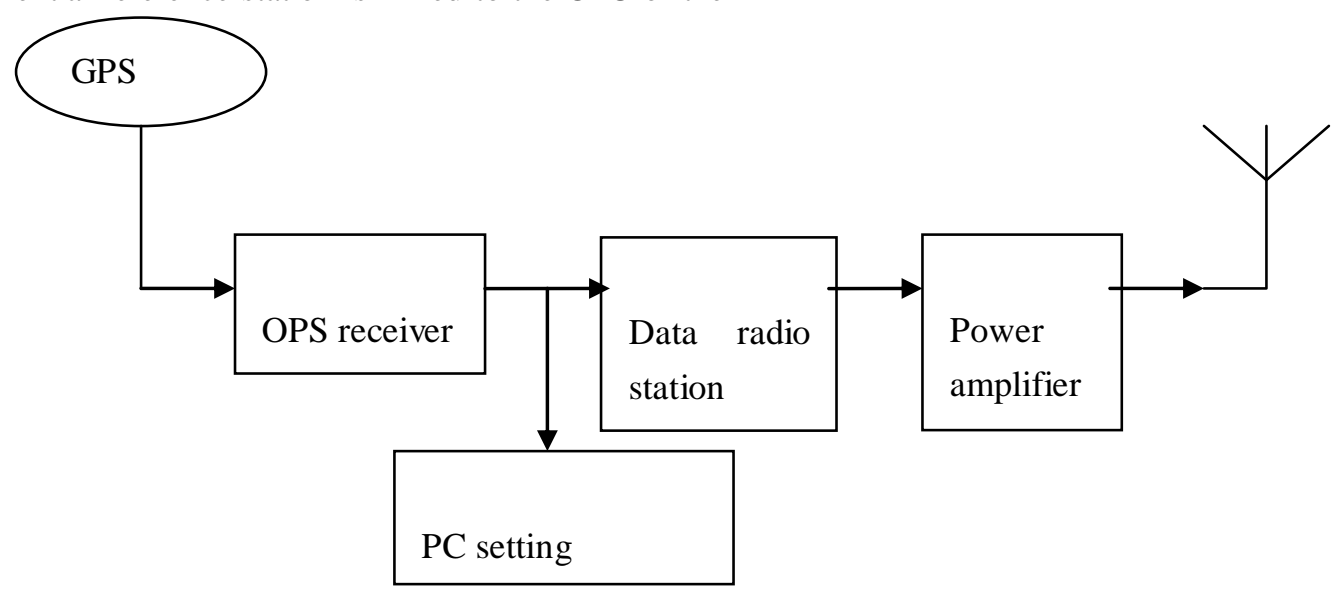

Figure 1 .Differential GPS base station configuration ground data center by RS232 gorge. As shown in figure .1.GPS module is connected with the antenna, providing the differential information to ground data station which will forward it to MDS after receiving the differential information.

\section{Main EVAluation METHOD OF THE EMBEDDED}

\section{SOFTWARE TESTING}

Coverage testing is the main method of AQ software white-box testing. As is known to all that in a software product, if there is some code has never been carried out, then this software is not reliable. There may be bug capable of crashing the whole software existing in the codes never been carried out. The purpose of coverage testing is to make all the codes be carried out at least for one time and try to spot the hidden bug during the testing and to ensure the software reliability to some degree. The coverage rate is the criteria for evaluating the coverage testing. The higher coverage is, the better testing result is. High enough coverage rate can enhance the confidence of the tester for the software .Coverage rate refers to the percentage of the program carried out during the testing in the total program. Coverage rate is calculated according to the following formula:

$$
\text { Coverage rate }=\frac{\text { Quantity of the program carried out }}{\text { otal program can be carried out in the program }} * 100 \%
$$

\section{WORKING PRINCIPLE OF RTRT UNIT TESTING}

Rational Test Real Time(RTRT) is a code testing tool provided by IBM Rational, especially designed for embedded, real-time or other commercial software product code staff. It can be pre-emptive debugging, so as to repair it before crashing.

Due to the powerful function, RTRT has complex working principle. Unit testing which is the most important start stage in the whole testing is the lowest level of testing activities in software development. So it's necessary to know how RTRT carries on unit testing and analyze its working principle.
It requires to set up auxiliary test module in unit testing. There are two kinds of auxiliary test module: one is testing driver, which is used to simulate the superior module to be tested. It receives test data in a unit test and deliver the related data to the tested module and then start the tested module and output corresponding results. The other is stub module, which is used for stimulating the module invoked during the work of the tested module. The stub module is invoked by tested module and usually only carry on a few data processing, so that the testers can check the interface of the tested module and its lower level module. Following is the diagram of general principle of unit testing: 


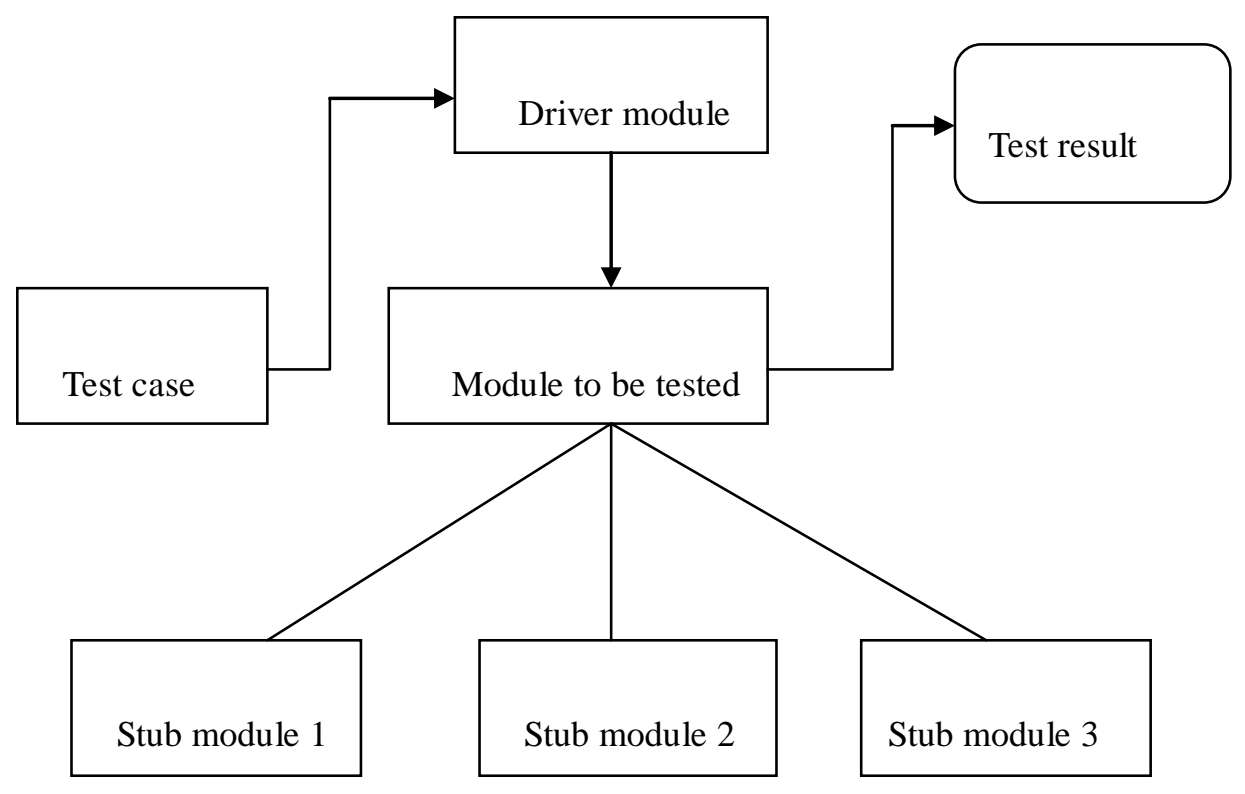

Figure 2.Unit testing environment

\section{CONCLUSION}

Set up the testing environment suitable for electronic system software according to the characteristics of the embedded software electronic system. Put forward the method and technology which can effectively test this system software considering the characteristics of the system. Improve the traditional software development testing $\mathrm{W}$ model to make it more suitable for the actual testing. Design and implement the specific testing. According to the actual needs of the testing object to vehicle electronic system, make full use of the existing software testing theory and method, extract suitable testing model and method, design a whole set of testing plan and implement testing according to this plan.

In this way, it achieved the software and hardware design of the ground data center. Form the data interaction control center of ground and engine by integrated application of embedded ARM chips, GPS module and data radio station and adoption of $\mathrm{pC} / 05-\mathrm{H}$ operating system to plan each task. Besides, formulate a series of communication protocols to jointly complete data interaction.

\section{REFERENCES}

[1] Stamoulis C, Betensky R A. A novel signal processing approach for the detection of copy number variations in the human genome[J]. Bioinformatics, 2012, 27(17):: 2338-2345.
[2] Mallat, Sté, and phane. A Wavelet Tour of Signal Processing. Elsevier LTD, Oxford, 2009:xxii+805.

[3] Strauss, W. "Digital signal processing." IEEE Signal Processing Magazine 17(2000):52-56.

[4] Ieee Acoustics, S. S. P. G. (1980). IEEE transactions on acoustics, speech, and signal processing. Signal Processing, 2(4), 199-200.

[5] Mallat, Sté, \& phane. (2009). A Wavelet Tour of Signal Processing. Elsevier LTD, Oxford.

[6] Krim, H., and M. Viberg. "Two decades of array signal processing research: the parametric approach." IEEE Signal Processing Magazine 13.4(1996):67 - 94.

[7] Stamoulis, C, and R. A. Betensky. "A novel signal processing approach for the detection of copy number variations in the human genome." Bioinformatics 27.17(2012):: 2338-2345.

[8] IEEE Acoustics, Speech, Signal Processing Group. "IEEE transactions on acoustics, speech, and signal processing." Signal Processing 2.4(1980): 199-200.

[9] White, S. A. (1989). Applications of distributed arithmetic to digital signal processing: a tutorial review. Assp Magazine IEEE, 6(3), 4-19.

[10] Krim, H., \& Viberg, M. (1996). Two decades ofarraysignalprocessing research: the parametric approach. IEEE Signal Processing Magazine, 13(4), 67 - 94.

[11] Stamoulis, C., \& Betensky, R. A. (2012). A novel signal processing approach for the detection of copy number variations in the human genome. Bioinformatics, 27(17), : 2338-2345.

[12] White, Stanley A. "Applications of distributed arithmetic to digital signal processing: a tutorial review." Assp Magazine IEEE 6.3(1989):4-19. 